İzzet Erdal*, Songül S. Yalçin*, Ayşegül Aksan, Didem Gençal and Nuray Kanbur

\title{
How useful are anthropometric measurements as predictive markers for elevated blood pressure in adolescents in different gender?
}

https://doi.org/10.1515/jpem-2020-0175

Received November 26, 2019; accepted June 30, 2020; published online August 24, 2020

\begin{abstract}
Background: Obesity and obesity-related diseases are one of the common health problems worldwide and have also been proposed to be important predictors of blood pressure and cardiovascular disease risk. The aim of our study is to determine the utility of different anthropometric measurements (body mass index [BMI], skinfold thickness [SFT], waist circumference [WC], mid-upper arm circumference [MUAC], arm circumference-height ratio [AHtR], and waist circumference-height ratio [WHtR]) as markers of hypertension (HT) risk in adolescents.

Methods: In this cross-sectional study, 544 participants aged between 12 and 13 years were included. Anthropometric measurements and blood pressure of participants were measured. The association of different anthropometric measurements with blood pressure was evaluated. Results: The frequency of both elevated blood pressure and HT was $30.2 \%$. Biceps, triceps, and suprailiac SFT have an impact on HT in girls but only suprailiac SFT in boys. WC measurements above the 85th percentile were strongly correlated with HT conditions, and this relationship was stronger in boys than in girls (3.3 vs. 2.6 fold). MUAC, WHtR, and AHtR measurements also have strong correlation with HT in boys but only WHtR has a poor relation in girls. In boys and girls with obesity, there was a positive association between obesity and blood pressures.
\end{abstract}

\footnotetext{
*Corresponding authors: İzzet Erdal, Division of Social Pediatrics, Department of Pediatrics, Faculty of Medicine, Hacettepe University, Ankara, Turkey. Tel.: +903123051133, Fax: +903123243284, E-mail: izzet.erdal@gmail.com. https://orcid.org/0000-0001-76452829; and Songül S. Yalçın, Department of Pediatrics, Faculty of Medicine, Hacettepe University, Ankara, Turkey. Tel.: +903123051133, Fax: +903123243284, E-mail: siyalcin@hacettepe.edu.tr

Ayşegül Aksan, Obesity Center DGD Clinics Sachsenhausen, Institute of Pharmaceutical Chemistry, Goethe University Frankfurt, Frankfurt, Germany

Didem Gençal, Şişli Hamidiye Etfal Training and Research Hospital, İstanbul, Turkey

Nuray Kanbur, Division of Adolescent Medicine, Department of Pediatrics, Faculty of Medicine, Hacettepe University, Ankara, Turkey
}

Conclusions: Not only age-related BMI z scores but also a number of other anthropometric measurements, such as WC, SFT, MUAC, WHtR, and AHtR, could have an influence on high blood pressure. The influence changes with gender during adolescence.

Keywords: adolescence; anthropometric measurement; blood pressure; body mass index; gender.

\section{Introduction}

Children and adolescents with obesity increased 10-fold between 1975 and 2016 [1]. The World Health Organization (WHO) predicts that by 2022, a greater number of children and adolescents will be affected by obesity than by underweight. Children with obesity are at an increased risk of developing concomitant diseases such as hypertension (HT), dyslipidemia, hyperglycemia, insulin resistance, nonalcoholic fatty liver, decreased renal function, and early atherosclerosis than healthy children of the same age group [2-7]. HT is a chronic condition associated with short- and long-term organ damage. Since its consequences are largely permanent but also preventable, early detection and prompt treatment of HT in children and adolescents play a key role in reducing associated risks and long-term effects [8-11]. Elevated blood pressure (EBP) has also been shown to be associated with target organ damage [12]. Studies have shown the average systemic blood pressure in children to have risen over recent years, and this increase is more pronounced in obese children. Although a global meta-analysis including 55 studies with a total population of over 120,000 children and adolescents aged 10-19 years showed no increase in the prevalence of HT up to 2013, studies evaluating the same data calculated HT rates of up to $26.8 \%$ in adolescents living in European countries [13]. There are limited and local data on the prevalence of HT in children and adolescents in Turkey [14, 15]. In Turkey, the Ministry of Health's 2014 nutrition and health status report noted a gradual increase in overweight and obesity among children and adolescents and stated that the prevalence of HT is expected to rise accordingly [16]. 
Due to the obesity epidemic, there is a growing need for preventive measures in children who are at increased risk of developing HT during childhood or later in life. Screening programs to detect EBP or HT in children and adolescents represent a useful strategy on which to base targeted interventions. However, in many cases, it remains difficult to predict which children are at risk for HT and to decide which anthropometric evaluations are appropriate at different stages of growth in childhood and adolescence. For decades, the standard assessments for children's growth have been based mainly on height and weight. Height has already been accepted as part of the definition of normative blood pressure values, along with age and gender.

Body mass index (BMI) is an essential anthropometric parameter used to predict body fat mass and show the possible risks for chronic diseases, including high blood pressure [17, 18]. Additional anthropometric measurements, such as skinfold thickness (SFT), waist circumference (WC), and waist circumference-height ratio (WHtR), have also been proposed to be important predictors of blood pressure and cardiovascular disease risk [2, 19-22]. On the other hand, the physical composition of the body in prepubertal boys and girls is generally similar. However, it undergoes important changes during puberty. Weight changes, particularly during pubertal maturation, reflect gender-specific changes, especially with regard to lean body mass and the proportion of body fat. However, BMI neither distinguishes between increased muscle mass and increased fat mass nor does it reflect the distribution of fat mass within the body [23]. A meta-analysis of five studies evaluating children aged between 2 and 19 years reported that BMI, WC, and WHtR were powerful anthropometric measures of body fat ratio [19]. Furthermore, on the basis of a study of 1197 adolescents, Pelegrini et al [20] reported that BMI, WC, and WHtR were also useful parameters for the follow-up of body fat ratio. In an anthropometric study conducted by Kavak et al [24] that included 597 male and 521 female Turkish adolescents aged between 10 and 15 years, BMI, WC, and WHtR ratio were found to be positively correlated with body fat ratio. However, in a study conducted with 1444 participants, childhood BMI values were reported to be a better indicator of the risk of adult HT than other anthropometric measurement methods such as WC and WHtR [25]. In another meta-analysis involving 30 studies, WC was said to have no superiority over BMI in showing the risk of cardiovascular disease [26]. In addition, a number of studies have reported that SFT measurements can be used to assess the body fat ratio by quantifying subcutaneous fat tissue $[27,28]$. Therefore, there is a need for investigations for the value of other anthropometric measurements such as midupper arm circumference (MUAC), WC, SFT, and WHtR, which are predictors of body fat ratio and regional fat mass, on blood pressure, the risk of cardiovascular disease [29-33].

The aim of our study was to determine and compare the utility of different types of anthropometric measurements as markers of HT risk and as descriptive parameters for the relationship between obesity and HT rates in adolescents aged between 12 and 13 years.

\section{Materials and methods}

The study was carried out between December 2017 and March 2018 in a school setting, following its approval by the Hacettepe University Ethics Committee (GO-17/837) and the Ankara Provincial National Education Directorate. Information letters were sent to the families of seventh- and eighth-grade students, and written informed consent was obtained from the participating children and their families before enrollment in the study. School visits were scheduled in consultation with the school administration in order to minimize any disruptive effects on the students' schoolwork and class attendance. At each visit, the investigator documented the participant's age, gender, medical history of chronic illness, current medication, and any occurrence of urinary tract infection in a semistructured questionnaire. Exclusion criteria include any renal or cardiac diseases.

OpenEpi (version 3.01) [34] calculated the sample size with a confidence level of $95 \%$, power of $80 \%$, the ratio of controls to cases of $25 \%$, hypothetical proportion of controls with exposure of $20 \%$, and odds ratio (OR) of 2.0 or greater. The Fleiss method revealed sample size as 553 persons.

\section{Anthropometric measurements}

The children's weight was measured, without shoes or heavy clothes, to the nearest $0.1 \mathrm{~kg}$ using a Seca digital scale (Seca 767 digital medical scale, Hamburg, Germany), and their height was measured to the nearest $0.1 \mathrm{~cm}$ using a stadiometer (Seca 220 telescopic height measuring rod, Hamburg, Germany). SFT (biceps, triceps, and suprailiac) was determined by means of skinfold calipers (Holtain skinfold calipers, Holtain Ltd, Crymych, UK). In addition, a stretchresistant tape measure was used to assess WC and MUAC. Measurements were taken at least twice for each location, and the average of the two was documented as the final value. If readings differed by more than $10 \%$, an additional measurement was carried out.

WHtR was calculated as WC/height (cm) and arm circumferenceheight ratio (AHtR) as MUAC/height $(\mathrm{cm})$.

Given neither global nor national reference values for these anthropometric measurements in adolescence [27, 28], participants whose measurements for MUAC, WC, SFT, WHtR, and AHtR were in the 85th percentile or higher in the percentile distributions of all the respective baseline values obtained in the study were classified as the "high measurement group".

Age-related height $z$-scores (HAZ), BMI (weight divided by height squared), and age-related BMI $z$-scores (BAZ) were calculated using the WHO-Antro Plus database [35] and categorized in groups as 
" $2 \geq B A Z>1$ " as overweight and "BAZ $>2$ " as obesity according to the definition of the WHO.

\section{Blood pressure measurements}

Blood pressure was measured using an electronic blood pressure monitor (Omron MIT Elite plus, Japan). Measurements were taken twice at $5 \mathrm{~min}$ intervals after the subjects had rested for $10 \mathrm{~min}$ in a seated position, and the mean of the two values was used. During the measurement, participants were seated with their back supported and their feet uncrossed on the floor. Finally, blood pressure values were classified as "normal", "EBP”, or "HT". "Normal blood pressure" was defined as systolic blood pressure and diastolic blood pressure values $<90$ th percentile, "elevated blood pressure" (now used instead of the term "prehypertension") was defined as systolic blood pressure and/ or diastolic blood pressure $\geq 90$ th percentile and $<95$ th percentile, and "HT" was defined as systolic blood pressure and/or diastolic blood pressure $\geq 95$ th percentile (on the basis of age, sex, and height percentiles published by the American Academy of Pediatrics) [36].

\section{Statistical analysis}

The Statistical Package for Social Sciences (SPSS Inc., Chicago, IL, USA), version 23.0, was used for all analyses. The distribution of variables was analyzed by the Kolmogrow-Smirnow test. Most anthropometric data had skewed distribution. Therefore, to evaluate anthropometric data including WC, MUAC, biceps SFT, triceps SFT, suprailiac SFT, WHtR, and AHtR, cases were classified into two groups as having normal or high values (85th percentile or higher). For risk factor assessment, the chi-square test was used to analyze the effects of a "one $z$-score deviation from HAZ and BAZ" and "high anthropometric measurements above the 85th percentile" on EBP or HT rates. Changes in the rates of EBP or HT were assessed by logistic regression in subgroups for each variable, and the ORs and 95\% confidence intervals (CIs) were calculated.

Multiple logistic regression analyses were performed to detect the influence of elevated anthropometric measurements on EBP or HT after controlling for age, gender, and HAZ of the children, as well as for the parents' age and the presence of any chronic diseases in the parents.

Receiver operating characteristic (ROC) curve analysis was used to calculate discriminative abilities and cutoff points of anthropometric measurements in relation to HT. The area under curve with $95 \%$ CI was calculated.

The relationship between blood pressure levels and the anthropometric measurements was analyzed by the Spearman correlation coefficient. Statistical significance was defined as a p value below 0.05 .

\section{Results}

\section{Study population characteristics}

Of 655 enrolled students, 111 children were excluded: 75 due to a history of chronic illness that could lead to high blood pressure, 29 due to recurrent urinary tract infections, and seven because they were 14 years or older. The remaining 544 students were included in the study. The characteristics of the study cohort are shown in Table 1.

\section{Frequencies of EBP and $\mathrm{HT}$ according to gender}

Figure 1 shows the respective proportions of students who were found to have systolic, diastolic, and general (systolic and/or diastolic) EBP, stage $1 \mathrm{HT}$, and stage $2 \mathrm{HT}$. The rate of high blood pressure (EBP and HT) was 30.2\% in the study population, and the rates were 28.4 and $31.9 \%$ for male and female students, respectively. No statistically significant difference was found between girls and boys in terms of EBP or HT (p>0.05) (Figure 1).

\section{Anthropometric measurements and blood pressure parameters}

The frequencies of high anthropometric measurements on both EBP and HT are given in Suppl Table 1.

The rates of systolic EBP were found to be higher among students with MUAC, AHtR, and WC above 85th

Table 1: Baseline characteristics*.

\begin{tabular}{lrrr}
\hline & Boys & Girls & Total \\
\hline $\mathrm{N}(\%)$ & $268(49.2)$ & $276(50.8)$ & 544 \\
Age, year & $13.09 \pm 0.58$ & $13.00 \pm 0.55$ & $13.04 \pm 0.57$ \\
Anthropometric & & & \\
$\quad$ measurements & & & \\
Weight (kg) & $53.3 \pm 13.6$ & $52.5 \pm 11.2$ & $52.9 \pm 12.4$ \\
Height (cm) & $159.2 \pm 8.8$ & $158.2 \pm 6.0$ & $158.7 \pm 7.5$ \\
BMI (kg/m $)$ & $20.9 \pm 4.2$ & $20.9 \pm 3.9$ & $20.9 \pm 4.1$ \\
WC (cm) & $73.5 \pm 11.2$ & $70.1 \pm 9.1$ & $71.8 \pm 10.3$ \\
MUAC (cm) & $23.5 \pm 3.9$ & $23.2 \pm 3.5$ & $23.4 \pm 3.7$ \\
Biceps SFT (mm) & $10.0 \pm 5.2$ & $10.3 \pm 4.3$ & $10.1 \pm 4.8$ \\
Triceps SFT (mm) & $13.5 \pm 5.6$ & $15.0 \pm 5.7$ & $14.3 \pm 5.7$ \\
Suprailiac SFT (mm) & $14.5 \pm 6.5$ & $14.4 \pm 5.2$ & $14.5 \pm 5.9$ \\
WHtR & $0.46 \pm 0.07$ & $0.44 \pm 0.06$ & $0.45 \pm 0.06$ \\
AHtR & $0.15 \pm 0.02$ & $0.15 \pm 0.02$ & $0.15 \pm 0.02$ \\
BAZ & $0.64 \pm 1.30$ & $0.50 \pm 1.19$ & $0.57 \pm 1.24$ \\
Nutritional status (\%) & & & \\
$\quad$ Low or normal weight & 57.8 & 65.2 & 61.6 \\
Overweight & 25.4 & 25.4 & 25.4 \\
Obese & 16.8 & 9.4 & 13.1 \\
Blood pressure (mmHg) & & & \\
Systolic BP & $110.7 \pm 9.4$ & $111.8 \pm 9.5$ & $111.3 \pm 9.4$ \\
Diastolic BP & $66.1 \pm 9.5$ & $69.1 \pm 9.3$ & $67.6 \pm 9.5$ \\
\hline N & & &
\end{tabular}

$\mathrm{N}$, number; BMI, body mass index; WC, waist circumference; MUAC, midupper arm circumference; SFT, skinfold thickness; WHtR, waist circumference-height ratio; AHtR, arm circumference-to-height ratio; BAZ, body mass index-for-age $z$ score; BP, blood pressure.

"Mean \pm SD. 


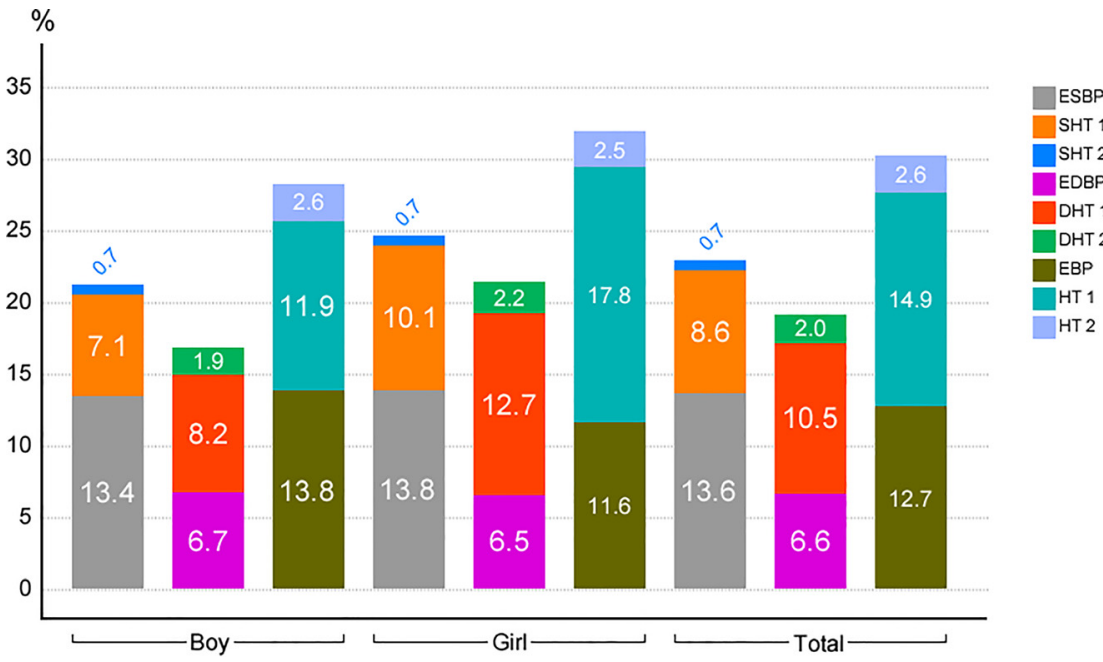

Figure 1: Normal blood pressure, elevated blood pressure, and hypertension frequencies according to gender. ESBP, elevated systolic blood pressure; SHT1, stage 1 systolic hypertension; SHT2, stage 2 systolic hypertension; EDBP, elevated diastolic blood pressure; DHT1, stage 1 diastolic hypertension; DHT2, stage 2 diastolic hypertension; EBP, elevated blood pressure; HT1, stage 1 hypertension; HT2, stage 2 hypertension. percentile (3.4 [1.9-6.1], 2.8 [1.5-5.1], and 2.7 [1.5-4.9], respectively) (Table 2). Students whose WHtR, WC, suprailiac SFT and MUAC measurements were above the 85th percentile had more than three-fold increased risk of having systolic hypertension [5.4 (2.8-10.2), 4.8 (\%95 CI=2.59.0), $4.6(2.4-8.7) 4.3$ fold (\%95 CI=2.2-8.3)].

The students with high MUAC and high AHtR had elevated ORs in both diastolic EBP and diastolic HT.

Besides HAZ, all measured anthropometric measurements had an impact on HT. Students with suprailiac SFT and MUAC and WHtR above 85th percentile had 3.7 fold (95\% CI=2.1-6.3), 3.4 fold (95\% CI=1.9-6.0), and 3.0 (1.7-5.4) increased OR for having systolic EBP and systolic HT, respectively.

Compared to BAZ values $\leq 1 z$ score, obese students (BAZ values $>2 z$ score) were associated with at least twofold OR for all blood pressure parameters (Table 2).

\section{Associations between anthropometric parameters and blood pressure by multiple logistic regression analysis according to gender}

Associations between the assessed anthropometric measurements and blood pressure readings were examined by multiple logistic regression analysis, adjusted for the participant's age, HAZ values, and maternal and paternal ages (Table 3). WC measurements above the 85th percentile were strongly associated with HT conditions (3.3 fold increased risk for boys, 2.6 fold increased risk for girls). Participants with an MUAC value above the 85 th percentile had a greater incidence of EBP and HT, with the exception of diastolic EBP in boys. However, in girls, there was only a weak association with EBP. The ratios of having EBP, HT, diastolic HT, and systolic EBP were increased in girls with a biceps SFT above the 85th

Table 2: High anthropometric measurements and elevated BPs, univariate analysis.

\begin{tabular}{|c|c|c|c|c|c|c|c|c|c|c|c|c|c|}
\hline & & \multicolumn{2}{|c|}{ SEBP vs. N } & \multicolumn{2}{|r|}{ SHT vs. N } & \multicolumn{2}{|c|}{ DEBP vs. N } & \multicolumn{2}{|r|}{ DHT vs. $\mathbf{N}$} & \multicolumn{2}{|r|}{ EBP vs N } & \multicolumn{2}{|r|}{ HT vs. N } \\
\hline & & OR & $95 \% \mathrm{Cl}$ & OR & $95 \% \mathrm{Cl}$ & OR & $95 \% \mathrm{Cl}$ & OR & $95 \% \mathrm{Cl}$ & OR & $95 \% \mathrm{Cl}$ & OR & $95 \% \mathrm{Cl}$ \\
\hline WC & $\leq 85 p$ vs. $>85 p$ & 2.7 & $1.5-4.9$ & 4.8 & $2.5-9.0$ & 1.6 & $0.7-3.7$ & 1.7 & $0.9-0.32$ & 3.1 & $1.7-5.7$ & 2.9 & $1.7-5.0$ \\
\hline MUAC & $\leq 85 p$ vs. $>85 p$ & 3.4 & $1.9-6.1$ & 4.3 & $2.2-8.3$ & 2.8 & $1.3-6.2$ & 2.8 & $1.6-5.2$ & 4.2 & $2.3-7.8$ & 3.4 & $1.9-6.0$ \\
\hline Biceps SFT & $\leq 85 p$ vs. $>85 p$ & 2.1 & $1.2-3.7$ & 1.8 & $0.9-3.7$ & 1.4 & $0.6-3.3$ & 2.2 & $1.2-4.0$ & 2.5 & $1.4-4.6$ & 2.7 & $1.6-4.6$ \\
\hline Triceps SFT & $\leq 85 p$ vs. $>85 p$ & 1.8 & $0.9-3.3$ & 2.9 & $1.5-5.6$ & 1.7 & $0.8-3.9$ & 2.2 & $1.2-3.9$ & 2.1 & $1.1-3.9$ & 2.5 & $1.4-4.3$ \\
\hline Suprailiac SFT & $\leq 85 p$ vs. $>85 p$ & 2.0 & $1.0-3.7$ & 4.6 & $2.4-8.7$ & 1.7 & $0.7-4.0$ & 2.7 & $1.5-4.8$ & 2.4 & $1.3-4.7$ & 3.7 & $2.1-6.3$ \\
\hline WHtR & $\leq 85 p$ vs. $>85 p$ & 2.5 & $1.3-4.6$ & 5.4 & $2.8-10.2$ & 2.2 & $1.0-4.8$ & 1.7 & $0.9-3.2$ & 3.7 & $2.0-6.8$ & 3.0 & $1.7-5.4$ \\
\hline AHtR & $\leq 85 p$ vs. $>85 p$ & 2.8 & $1.5-5.1$ & 3.7 & $1.9-7.2$ & 2.3 & $1.0-5.2$ & 2.3 & $1.3-4.3$ & 3.7 & $2.0-6.8$ & 2.9 & $1.6-5.1$ \\
\hline \multirow[t]{2}{*}{ BAZ } & Overweight vs. $<1 \mathrm{z}$ & 1.9 & $1.1-3.3$ & 1.3 & $0.6-2.7$ & 0.6 & $0.2-1.5$ & 1.4 & $0.8-2.6$ & 1.8 & $1.0-3.3$ & 1.6 & $0.9-2.7$ \\
\hline & Obese vs. $<1 z$ & 2.4 & $1.2-4.8$ & 4.0 & $2.0-7.9$ & 2.5 & $1.1-5.7$ & 3.0 & $1.5-5.8$ & 3.5 & $1.7-7.2$ & 3.9 & $2.1-7.2$ \\
\hline
\end{tabular}

SEBP, systolic elevated blood pressure; SHT, systolic hypertension; DEBP, diastolic elevated blood pressure; DHT, diastolic hypertension; EBP, elevated blood pressure; HT, hypertension; WC, waist circumference; MUAC, midupper arm circumference; SFT, skinfold thickness; WHtR, waist circumference-height ratio; AHtR, arm circumference-to-height ratio; $\mathrm{BAZ}$, body mass index-for-age $z$ score; N, normal; >85p, measurements that are above the 85 percentiles; $\mathrm{OR}$, odds ratio; $\mathrm{Cl}$, confidence interval. 
percentile, and the highest one was found for $\mathrm{HT}(\mathrm{OR}=3.2$, $95 \% \mathrm{CI}=1.5-7.1)$. A triceps SFT above the 85th percentile was associated with greater odds of systolic HT (OR=3.4, 95\% $\mathrm{CI}=1.4-8.1$ ), diastolic HT (OR=3.2, 95\% $\mathrm{CI}=1.4-7.2)$, EBP (OR=2.6, 95\% CI=1.0-6.4), and HT (OR=3.5, 95\% CI=1.6-7.5) in girls. No association was found between triceps or biceps SFT and blood pressure in boys. The association of blood pressure and suprailiac SFT was found in boys and girls (for HT, 4.4-fold for boys and 3.2-fold for girls). Boys with overweight had a 3.4-fold ( $95 \% \mathrm{CI}=1.4-8.1)$ increased probability of systolic EBP compared to boys of normal weight. In boys with obesity, all blood pressure measurements except diastolic EBP were associated with an increased probability of high blood pressure, while this association was strongest in systolic HT (OR=5.0, 95\% CI=1.4-17.4). No increased risk for EBP or HT was observed in girls with overweight. In girls with obesity, there was a positive association between obesity and systolic HT and obesity and HT.

\section{Ability of anthropometric parameters to discriminate high blood pressure}

Females had small area under curve for WC and WHtR which is below than 0.60 . However, WC revealed the highest area under curve for males. Females had lower estimates than males. Most anthropometric parameters showed poor discriminative ability (between 0.60 and 0.70 ) for HT. Cutoff points showed difference for biceps SFT between genders ( $8 \mathrm{~mm}$ for boys, $12.1 \mathrm{~mm}$ for girls) (Table 4).

\section{Correlation analysis between anthropometric measurements and blood pressure values by gender}

The correlation analysis between blood pressure and anthropometric measurements is shown in Table 5. All anthropometric measurements investigated in the study were found to correlate with systolic blood pressure and, to a lesser extent, diastolic blood pressure. In the entire study population, regardless of gender, the strongest correlation between systolic blood pressure and anthropometric measurements was shown for BAZ $(r=0.35, p<0.01)$. In boys, the strongest correlation with systolic blood pressure was demonstrated for WC $(r=0.43, p<0.01)$ followed by MUAC $(r=0.42, p<0.01)$, whereas BAZ had the strongest correlation in girls $(r=0.33, p<0.01)$. The correlation between diastolic blood pressure and anthropometric

Table 3: High anthropometric measurements and elevated BPs in boys and girls, multivariate analysis*.

\begin{tabular}{|c|c|c|c|c|c|c|c|c|c|c|c|c|c|}
\hline & & \multicolumn{2}{|r|}{ SEBP vs. N } & \multicolumn{2}{|r|}{ DEBP vs. N } & \multicolumn{2}{|r|}{ EBP vs. N } & \multicolumn{2}{|r|}{ SHT vs. N } & \multicolumn{2}{|r|}{ DHT vs. N } & \multicolumn{2}{|r|}{ HT vs. N } \\
\hline & & OR & $95 \% \mathrm{Cl}$ & OR & $95 \% \mathrm{Cl}$ & OR & $95 \% \mathrm{Cl}$ & OR & $95 \% \mathrm{Cl}$ & OR & $95 \% \mathrm{Cl}$ & OR & $95 \% \mathrm{Cl}$ \\
\hline \multicolumn{14}{|l|}{ Boys } \\
\hline WC & $\leq 85 p$ vs. $>85 p$ & 1.8 & $0.6-5.4$ & 4.8 & $1.6-14.6$ & 1.0 & $0.3-3.8$ & 2.2 & $0.9-5.7$ & 3.00 & $1.2-7.3$ & 3.3 & $1.4-7.9$ \\
\hline MUAC & $\leq 85 p$ vs. $>85 p$ & 4.2 & $1.7-10.4$ & 7.8 & $2.3-26.5$ & 1.7 & $0.5-6.3$ & 4.0 & $1.5-10.3$ & 3.5 & $1.3-9.4$ & 5.1 & $2.1-12.9$ \\
\hline Biceps SFT & $\leq 85 p$ vs. $>85 p$ & 2.0 & $0.8-4.8$ & 1.4 & $0.5-4.3$ & 1.4 & $0.4-5.3$ & 1.8 & $0.7-4.7$ & 2.3 & $0.9-6.0$ & 2.1 & $0.9-4.8$ \\
\hline Triceps SFT & $\leq 85 p$ vs. $>85 p$ & 1.3 & $0.4-3.7$ & 1.7 & $0.5-5.3$ & 1.1 & $0.2-5.1$ & 1.1 & $0.4-3.4$ & 1.6 & $0.5-4.5$ & 1.2 & $0.5-3.3$ \\
\hline Suprailiac SFT & $\leq 85 p$ vs. $>85 p$ & 2.1 & $0.8-5.5$ & 6.1 & $2.0-18.8$ & 0.8 & $0.2-3.7$ & 3.2 & $1.3-8.1$ & 2.0 & $0.7-5.3$ & 4.4 & $1.8-10.5$ \\
\hline WHtR & $\leq 85 p$ vs. $>85 p$ & 3.6 & $1.5-9.0$ & 5.1 & $1.7-15.2$ & 1.1 & $0.3-4.3$ & 2.8 & $1.1-7.1$ & 4.2 & $1.6-10.6$ & 3.8 & $1.6-9.3$ \\
\hline $\mathrm{AHtR}$ & $\leq 85 p$ vs. $>85 p$ & 3.6 & $1.5-8.6$ & 7.8 & $2.6-23.4$ & 1.4 & $0.4-5.3$ & 3.8 & $1.5-9.3$ & 3.2 & $1.2-8.2$ & 5.2 & $2.2-12.1$ \\
\hline \multirow[t]{2}{*}{ BAZ } & Overweight vs. $<1 z$ & 3.4 & $1.4-8.1$ & 1.4 & $0.4-4.8$ & 0.6 & $0.2-2.6$ & 0.9 & $0.3-2.6$ & 2.5 & $1.0-6.2$ & 1.2 & $0.5-3.0$ \\
\hline & Obese vs. $<1 z$ & 3.9 & $1.3-11.4$ & 5.0 & $1.4-17.4$ & 0.7 & $0.1-3.5$ & 2.3 & $1.1-4.9$ & 3.0 & $1.0-9.1$ & 4.2 & $1.6-10.9$ \\
\hline \multicolumn{14}{|l|}{ Girls } \\
\hline WC & $\leq 85 p$ vs. $>85 p$ & 1.8 & $0.6-5.4$ & 4.8 & $1.8-13.0$ & 0.9 & $0.2-4.6$ & 0.9 & $0.3-2.8$ & 2.3 & $0.7-7.1$ & 2.6 & $1.0-6.7$ \\
\hline MUAC & $\leq 85 p$ vs. $>85 p$ & 1.7 & $0.7-4.6$ & 2.1 & $0.7-5.7$ & 2.8 & $0.7-10.6$ & 1.4 & $0.5-3.7$ & 3.9 & $1.4-11.1$ & 1.8 & $0.7-4.4$ \\
\hline Biceps SFT & $\leq 85 p$ vs. $>85 p$ & 2.1 & $0.9-5.0$ & 1.8 & $0.7-4.9$ & 1.0 & $0.3-4.0$ & 2.7 & $1.1-6.4$ & 2.7 & $1.0-7.1$ & 3.2 & $1.5-7.1$ \\
\hline Triceps SFT & $\leq 85 p$ vs. $>85 p$ & 2.0 & $0.8-4.6$ & 3.4 & $1.4-8.1$ & 2.2 & $0.7-7.1$ & 3.2 & $1.3-7.2$ & 2.6 & $1.0-6.4$ & 3.5 & $1.7-7.5$ \\
\hline Suprailiac SFT & $\leq 85 p$ vs. $>85 p$ & 1.2 & $0.4-3.7$ & 3.8 & $1.4-10.1$ & 2.1 & $0.5-8.5$ & 1.9 & $0.7-5.0$ & 1.9 & $0.6-6.2$ & 3.2 & $1.3-7.5$ \\
\hline WHtR & $\leq 85 p$ vs. $>85 p$ & 1.2 & $0.4-3.9$ & 6.4 & $2.3-17.8$ & 1.9 & $0.5-7.0$ & 1.1 & $0.8-1.8$ & 2.6 & $0.9-7.9$ & 2.6 & $1.0-6.7$ \\
\hline AHtR & $\leq 85 p$ vs. $>85 p$ & 1.5 & $0.6-4.0$ & 1.3 & $0.4-3.8$ & 2.2 & $0.6-8.1$ & 1.2 & $0.8-1.8$ & 3.8 & $1.4-10.4$ & 1.1 & $0.4-2.8$ \\
\hline \multirow[t]{2}{*}{ BAZ } & Overweight vs. $<1 \mathrm{z}$ & 1.1 & $0.5-2.5$ & 1.4 & $0.5-3.6$ & 0.7 & $0.1-3.3$ & 1.8 & $0.8-3.9$ & 1.7 & $0.7-4.2$ & 1.8 & $0.9-3.7$ \\
\hline & Obese vs. $<1 z$ & 0.9 & $0.2-3.7$ & 3.5 & $1.6-7.8$ & 2.1 & $0.8-5.6$ & 1.6 & $0.5-5.2$ & 3.4 & $0.9-12.2$ & 3.0 & $1.1-8.6$ \\
\hline
\end{tabular}

Statistically significant results are highlighted within in italics. SEBP, systolic elevated blood pressure; SHT, systolic hypertension; DEBP, diastolic elevated blood pressure; DHT, diastolic hypertension; EBP, elevated blood pressure; HT, hypertension; WC, waist circumference; MUAC, midupper arm circumference; SFT, skinfold thickness; WHtR, waist circumference-height ratio; AHtR, arm circumference-to-height ratio; $\mathrm{BAZ}$, body mass index-for-age $z$ score; $\mathrm{N}$, normal; $>85 \mathrm{p}$, measurements that are above the 85 percentiles; OR, odds ratio; $\mathrm{Cl}$, confidence interval. *Adjusting age of the participant, HAZ values, maternal and paternal ages. 
Table 4: Discriminative abilities and optimal cutoff points of antropometric measurements in relation to systolic and/or diastolic hypertension.

\begin{tabular}{|c|c|c|c|c|c|c|c|}
\hline & & Area under curve & Asymptotic $95 \% \mathrm{Cl}$ & Cutoff point & Sensitivity & Specificity & Youden index \\
\hline \multirow[t]{2}{*}{ WC } & Male & 0.66 & $0.58-0.73$ & 77.8 & 0.51 & 0.75 & 0.26 \\
\hline & Female & 0.58 & $0.51-0.66$ & 73.3 & 0.45 & 0.74 & 0.19 \\
\hline \multirow[t]{2}{*}{ MUAC } & Male & 0.65 & $0.58-0.73$ & 27.3 & 0.36 & 0.90 & 0.25 \\
\hline & Female & 0.61 & $0.54-0.69$ & 23.3 & 0.57 & 0.63 & 0.21 \\
\hline \multirow[t]{2}{*}{ Biceps SFT } & Male & 0.60 & $0.53-0.68$ & 8.0 & 0.74 & 0.44 & 0.17 \\
\hline & Female & 0.63 & $0.55-0.70$ & 12.1 & 0.43 & 0.82 & 0.25 \\
\hline \multirow[t]{2}{*}{ Triceps SFT } & Male & 0.62 & $0.55-0.69$ & 16.1 & 0.46 & 0.75 & 0.21 \\
\hline & Female & 0.62 & $0.55-0.70$ & 16.1 & 0.53 & 0.71 & 0.24 \\
\hline \multirow[t]{2}{*}{ Suprailiac SFT } & Male & 0.63 & $0.55-0.71$ & 17.4 & 0.53 & 0.74 & 0.27 \\
\hline & Female & 0.62 & $0.55-0.69$ & 17.4 & 0.45 & 0.77 & 0.22 \\
\hline \multirow[t]{2}{*}{ WHtR } & Male & 0.64 & $0.56-0.72$ & 0.47 & 0.59 & 0.67 & 0.26 \\
\hline & Female & 0.58 & $0.50-0.65$ & 0.47 & 0.38 & 0.81 & 0.19 \\
\hline \multirow[t]{2}{*}{ AHtR } & Male & 0.65 & $0.57-0.72$ & 0.17 & 0.38 & 0.88 & 0.26 \\
\hline & Female & 0.61 & $0.53-0.68$ & 0.15 & 0.46 & 0.75 & 0.21 \\
\hline \multirow[t]{2}{*}{$\mathrm{BAZ}$} & Male & 0.64 & $0.56-0.72$ & 1.78 & 0.43 & 0.86 & 0.29 \\
\hline & Female & 0.63 & $0.55-0.70$ & 1.40 & 0.40 & 0.80 & 0.19 \\
\hline
\end{tabular}

WC, waist circumference; MUAC, midupper arm circumference; SFT, skinfold thickness; WHtR, waist circumference-height ratio; AHtR, arm circumference-to-height ratio; BAZ, body mass index-for-age $z$ score.

measurements was below $\mathrm{r}=0.30$. The strongest correlations for diastolic blood pressure were found with triceps SFT $(r=0.24, p<0.01)$ in the total study population but for WC in males $(\mathrm{r}=0.26, \mathrm{p}<0.01)$ and for BAZ in females $(\mathrm{r}=0.27, \mathrm{p}<0.01)$.

\section{Discussion}

\section{Changes in HT frequency with BMI}

Our study, conducted in Turkish children aged 12 to 13 years, revealed more than one in 10 adolescents $(12.7 \%)$ had EBP and nearly one in five (17.5\%) had HT. This is in accordance with the higher prevalence of HT reported in some other European and Turkish studies [14, 15, 37]. In a survey conducted in Bursa, Turkey, including 1000 subjects aged 6-13 years, the proportion of children and adolescents with EBP and HT was reported to be $10.8 \%$ [14]. In another study performed in Sakarya including 2166 children and adolescents aged 6-15 years, overall combined EBP and HT rates were determined to be $30.4 \%$, while HT prevalence was found to be twice as high in children with obesity versus those without obesity [15]. Similarly, when BMI-based groups of overweight and obesity were taken, we determined an increased OR for obesity with systolic blood pressure (systolic EBP 2.4-fold, systolic HT 4.0-fold higher), diastolic blood pressure (diastolic EBP 2.5-fold, diastolic HT 3.0-fold higher), and overall systemic blood pressure (EBP 3.5-fold, HT 3.9-fold higher). However, overweight was associated only with systolic EBP but not with diastolic or systemic blood pressure values. After

Table 5: Correlation between blood pressures and anthropometric measurements, Spearman correlation analysis.

\begin{tabular}{|c|c|c|c|c|c|c|c|c|c|c|c|c|c|}
\hline & & SBP & DBP & Age & HAZ & BAZ & WC & MUAC & Biceps SFT & Triceps SFT & Suprailiac SFT & WHtR & AHtR \\
\hline \multirow[t]{3}{*}{ SBP } & Overall $(n=544)$ & & $0.69^{* *}$ & 0.05 & $0.24^{* *}$ & $0.35^{* *}$ & $0.34^{* *}$ & $0.35^{* *}$ & $0.26^{* *}$ & $0.28^{* *}$ & $0.33^{* *}$ & $0.27^{* *}$ & $0.30^{* *}$ \\
\hline & Boys $(n=268)$ & & $0.67^{* *}$ & $0.17^{* *}$ & $0.32^{* *}$ & $0.39^{* *}$ & $0.43^{* *}$ & $0.42^{* *}$ & $0.28^{* *}$ & $0.29^{* *}$ & $0.37^{* *}$ & $0.31^{* *}$ & $0.33^{* *}$ \\
\hline & Girls $(n=276)$ & & $0.72^{* *}$ & -0.05 & $0.16^{* *}$ & $0.33^{* *}$ & $0.28^{* *}$ & $0.29^{* \star}$ & $0.24^{* *}$ & $0.25^{* *}$ & $0.29^{* *}$ & $0.25^{* *}$ & $0.27^{\star *}$ \\
\hline \multirow[t]{3}{*}{ DBP } & Overall $(n=544)$ & $0.69^{* *}$ & & 0.03 & $0.14^{* *}$ & $0.21^{* *}$ & $0.19^{* *}$ & $0.21^{* \star}$ & $0.20^{* *}$ & $0.24^{* *}$ & $0.24^{* *}$ & $0.13^{* *}$ & $0.18^{* *}$ \\
\hline & Boys $(n=268)$ & $0.67^{* *}$ & & $0.17^{* *}$ & $0.20^{* *}$ & $0.18^{* *}$ & $0.26^{* *}$ & $0.23^{* *}$ & $0.20^{* *}$ & $0.23^{* *}$ & $0.24^{* *}$ & $0.16^{*}$ & $0.14^{*}$ \\
\hline & Girls $(n=276)$ & $0.72^{\star *}$ & & -0.08 & 0.09 & $0.27^{* *}$ & $0.16^{* *}$ & $0.22^{* *}$ & $0.19^{* *}$ & $0.21^{* *}$ & $0.24^{* *}$ & $0.14^{*}$ & $0.22^{* *}$ \\
\hline
\end{tabular}

${ }^{*} \mathrm{p}<0.05,{ }^{* *} \mathrm{p}<0.01$.

SBP, systolic blood pressure; DBP, diastolic blood pressure; HAZ, height-for-age $z$ score; BAZ, BMI-for-age $z$ score; WC, waist circumference; MUAC, midupper arm circumference; SFT, skinfold thickness; WHtR, waist circumference-height ratio; AHtR, arm circumference-to-height ratio; BMI, body mass index. 
adjusting for the age of the child, HAZ, and the mother's and father's ages, a positive association was found in male children between obesity and all blood pressure measurements except diastolic EBP and between overweight and systolic EBP. However, obesity in girls increased OR for systolic HT and HT. However, overweight had no impact on EBP or HT. Thus, in Turkish children, obesity was determined to be a risk factor in both genders. However, being overweight is a risk factor only for boys.

\section{Changes in HT frequency with other anthropometric evaluations according to gender}

In our study, WC measurements above the 85th percentile were strongly associated with the presence of HT, an association that was stronger in boys than in girls. The association between blood pressure and suprailiac SFT was also more pronounced in boys. Similarly, WHtR is a stronger predictor in boys than in girls. While WHtR causes more risk increase than WC in boys, this relationship was not observed in girls. Additionally, to detect HT, anthropometric parameters revealed gender differences in areas under ROC curves (Figure 2). These results suggest that the increase in abdominal adipose tissue as measured by WC, WHtR, and suprailiac SFT was more predictive for HT in boys than in girls. Gender-specific changes in fat distribution could be taken into consideration when managing HT with anthropometric parameters.

In adolescents, lean body mass constitutes approximately $80 \%$ of body weight in early puberty. This proportion changes to $75 \%$ in girls and $90 \%$ in boys at maturity. While lean body mass increases in both sexes, its proportion of total body weight decreases in girls, due to the greater increase in adipose mass, whereas it increases in boys, primarily as a result of increased muscle mass.
During puberty, the percentage of body fat in (normal weight) females increases from $15 \%$ in Tanner stage 1 to $27 \%$ in Tanner stage 4, whereas in males, it decreases from $14 \%$ in stage 1 to $11 \%$ in stage 2 and remains unchanged in stages 3, 4, and 5. Thus, during the course of adolescence, the relative mass of subcutaneous and abdominal fat tissues undergoes a natural, development-related increase in girls, while the opposite is true in boys $[38,39]$. Therefore, an increase in body fat percentage during puberty in girls has a normal physiological and developmental process, whereas in boys, it is not an appropriate developmental change and may, therefore, more accurately predict $\mathrm{HT}$ in boys.

Important gender-dominant developmental changes in body composition and shape during puberty include pelvic remodeling in girls, in which the female pelvis widens, and an increase in biacromial breadth (broadening of the shoulders) in boys [40, 41]. In the present study, suprailiac fat gain was found to be the only predictive SFT for HT in boys. No association was found between triceps or biceps SFT and blood pressure in boys. On the other hand, the prevalence of systolic HT, diastolic HT, EBP, and especially HT was increased in girls with a biceps or triceps SFT above the 85th percentile. In the literature, SFT has been well described as a more direct measure of adiposity and has been found to correlate well with body fat content in both children and adults [42]. In a study conducted in China, it was stated that the combination of BMI and SFT measurement was more powerful than BMI alone for risk assessment regarding relatively high blood pressure in children and adolescents [43]. However, our results suggest that the localization of subcutaneous fat mass measured by SFT is important and that the predictive value of SFT on HT differs in girls and boys.

MUAC is an anthropometric measurement that used to detect nutritional status. In recent years, it has also been used to predict nutritional status-related comorbidities. In our study, we found that MUAC and AHtR were associated
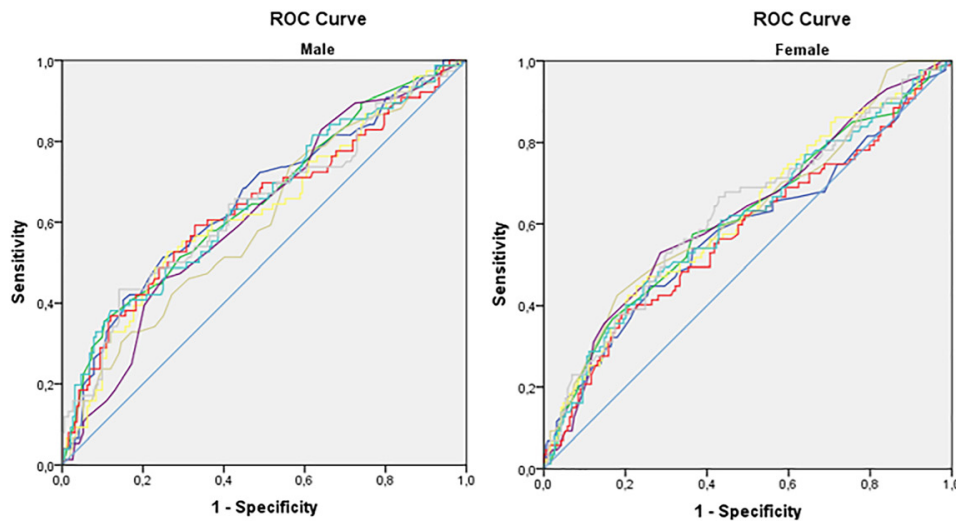

Figure 2: Receiver operating characteristic (ROC) curve and under curve areas of antropometric measurements in relation to systolic and/or diastolic hypertension. 
with increased risk for all high blood pressure conditions except isolated EBP in boys, while only diastolic HT was associated in girls. These results are compatible with previous studies and support that MUAC and AHtR can be used to screen high blood pressure [31, 32].

\section{Limitations and strengths}

The study has some limitations. Firstly, birth weight, a risk factor for obesity, has not been evaluated. Secondly, dietary salt intake, one of the risk factors for HT, has also not been analyzed. In addition, since the study was carried out as part of a school screening program, it was not possible to assess the participants' pubertal stage, an important factor affecting developmental changes in body fat mass. Strengths of the study include the fact that, although the research was conducted as a single-center study, the school accepts students from all over the city and it was representative of Ankara. Our study, for the first time, shows the association of different anthropometric measures with the risk of HT in a group of otherwise healthy adolescents with a high rate of obesity. All adolescents with a nonobesity-related health problem, known chronic disease, and recurrent urinary tract infections, as well as those using a medication, were excluded from the study.

\section{Conclusion}

Our data analysis shows that not only BMI but also a number of other anthropometric measurements such as WC, MUAC, WHtR, AHtR, and SFT had an impact on high blood pressure. Since the predictive power of anthropometric measurements varies with gender, the anthropometric measurements used in the assessment of adolescents' blood pressure should be determined according to gender. These measurements are reliable risk indicators that can be utilized as an alternative or in addition to BMI to predict the risk of obesity-related HT. In further studies, interactions between different anthropometric data and blood pressure could be evaluated in adolescence period from different developmental stages.

Acknowledgment: The authors thank Janet Collins, iCCC Rhein-Main, Frankfurt am Main, Germany, for her help in writing and proofreading the manuscript.

Research funding: None.

Author contributions: I.E. designed the study, collected the data, and drafted the manuscript. A.A. and D.G. collected the data. N.K. was involved in reviewing and editing of the manuscript. All authors approved the final manuscript. S.S.Y. designed and implemented the analyses and involved in reviewing and editing of the manuscript and did supervision.

Competing of interests: There are no conflicts of interest. Informed consent: Informed consent was obtained from all individuals included in this study.

Ethical approval: Research involving human subjects complied with all relevant national regulations, institutional policies, and is in accordance with the tenets of the Helsinki Declaration (as revised in 2013) and has been approved by the Hacettepe University Ethics Committee (GO-17/837).

\section{References}

1. Abarca-Gómez L, Abdeen ZA, Hamid ZA, Abu-Rmeileh NM, AcostaCazares B, Acuin C, et al. Worldwide trends in body-mass index, underweight, overweight, and obesity from 1975 to 2016: a pooled analysis of 2416 population-based measurement studies in 1289 million children, adolescents, and adults. Lancet 2017; 390:2627-42.

2. Bastien M, Poirier P, Lemieux I, Despres JP. Overview of epidemiology and contribution of obesity to cardiovascular disease. Prog Cardiovasc Dis 2014;56:369-81.

3. The Global Burden Of Diseases 2015 Obesity Collaborators. Health effects of overweight and obesity in 195 countries over 25 years. N Engl J Med 2017;377:13-27.

4. Lloyd LJ, Langley-Evans SC, McMullen S. Childhood obesity and risk of the adult metabolic syndrome: a systematic review. Int J Obes 2012;36:1-11.

5. World Health Organization. Obesity: preventing and managing the global epidemic. Geneva: World Health Organization; 2000.

6. Czernichow S, Kengne AP, Stamatakis E, Hamer M, Batty GD. Body mass index, waist circumference and waist-hip ratio: which is the better discriminator of cardiovascular disease mortality risk? Evidence from an individual-participant metaanalysis of 82864 participants from nine cohort studies. Obes Rev 2011;12:680-7.

7. Di Bonito P, Valerio G, Licenziati MR, Miraglia Del Giudice E, Baroni MG, Morandi A, et al. High uric acid, reduced glomerular filtration rate and non-alcoholic fatty liver in young people with obesity. J Endocrinol Invest 2020;43:461-8.

8. Baracco R, Kapur G, Mattoo T, Jain A, Valentini R, Ahmed M, et al. Prediction of primary vs secondary hypertension in children. J Clin Hypertens 2012;14:316-21.

9. Kapur G, Ahmed M, Pan C, Mitsnefes M, Chiang M, Mattoo TK. Secondary hypertension in overweight and stage 1 hypertensive children: a Midwest Pediatric Nephrology Consortium report. J Clin Hypertens 2010;12:34-9.

10. Rao G. Diagnosis, epidemiology, and management of hypertension in children. Pediatrics 2016;138:e20153616.

11. Flynn JT, Alderman MH. Characteristics of children with primary hypertension seen at a referral center. Pediatr Nephrol 2005;20: 961-6. 
12. Urbina EM, Khoury PR, McCoy C, Daniels SR, Kimball TR, Dolan LM. Cardiac and vascular consequences of pre-hypertension in youth. J Clin Hypertens 2011;13:332-42.

13. de Moraes AC, Lacerda MB, Moreno LA, Horta BL, Carvalho HB. Prevalence of high blood pressure in 122,053 adolescents: a systematic review and meta-regression. Medicine (Baltim) 2014;93: e232.

14. Demirci H, Nuhoglu C, Ursavas IS, Isildak S, Basaran EO, Kılıc MY. Obesity and asymptomatic hypertension among children aged 6 13 years living in Bursa, Turkey. Fam Pract 2013;30:629-33.

15. Önsüz FM, Demir F. Prevalence of hypertension and its association with obesity among schoolchildren aged 6-15 living in Sakarya Province in Turkey. Turk J Med Sci 2015;45:907-12.

16. Bakanlığı TS. Türkiye Beslenme ve Sağıı Araştırması 2010: Beslenme durumu ve alışkanlıklarının değerlendirilmesi sonuç raporu. Ankara: Sağlık Bakanlığı Sağlık Araştırmaları Genel Müdürlüğü; 2014.

17. Falkner B, Gidding SS, Ramirez-Garnica G, Wiltrout SA, West D, Rappaport EB. The relationship of body mass index and blood pressure in primary care pediatric patients. J Pediatr 2006;148: 195-200.

18. Sorof JM, Lai D, Turner J, Poffenbarger T, Portman RJ. Overweight, ethnicity, and the prevalence of hypertension in school-aged children. Pediatrics 2004;113:475-82.

19. Alves Junior CA, Mocellin MC, Gonçalves ECA, Silva DA, Trindade EB. Anthropometric indicators as body fat discriminators in children and adolescents: a systematic review and metaanalysis. Adv Nutr 2017;8:718-27.

20. Pelegrini A, Silva DA, Silva JM, Grigollo L, Petroski EL. [Anthropometric indicators of obesity in the prediction of high body fat in adolescents]. Rev Paul Pediatr 2015;33:56-62.

21. Giampietro O, Virgone E, Carneglia L, Griesi E, Calvi D, Matteucci E. Anthropometric indices of school children and familiar risk factors. Prev Med 2002;35:492-8.

22. Barba G, Troiano E, Russo P, Strazzullo P, Siani A. Body mass, fat distribution and blood pressure in Southern Italian children: results of the ARCA project. Nutr Metabol Cardiovasc Dis 2006;16:239-48.

23. Yajnik CS, Yudkin JS. The YY paradox. Lancet 2004;363:163.

24. Kavak V, Pilmane M, Kazoka D. Body mass index, waist circumference and waist-to-hip-ratio in the prediction of obesity in Turkish teenagers. Coll Antropol 2014;38:445-51.

25. Fan H, Zhu Q, Medrano-Gracia P, Zhang X. Comparison of child adiposity indices in prediction of hypertension in early adulthood. J Clin Hypertens 2019;21:1858-62.

26. Lo K, Wong M, Khalechelvam P, Tam W. Waist-to-height ratio, body mass index and waist circumference for screening paediatric cardio-metabolic risk factors: a meta-analysis. Obes Rev 2016;17:1258-75.

27. Freedman DS, Ogden CL, Blanck HM, Borrud LG, Dietz WH. The abilities of body mass index and skinfold thicknesses to identify children with low or elevated levels of dual-energy X-ray absorptiometry-determined body fatness. J Pediatr 2013;163:160-6 e1.

28. Silva DR, Ribeiro AS, Pavão FH, Ronque ER, Avelar A, Silva AM, et al. Validity of the methods to assess body fat in children and adolescents using multi-compartment models as the reference method: a systematic review. Rev Assoc Med Bras 1992;59:475-86.
29. McCarthy HD, Ashwell M. A study of central fatness using waistto-height ratios in UK children and adolescents over two decades supports the simple message-'keep your waist circumference to less than half your height'. Int J Obes 2006;30:988.

30. Kahn HS, Imperatore G, Cheng YJ. A population-based comparison of BMI percentiles and waist-to-height ratio for identifying cardiovascular risk in youth. J Pediatr 2005;146:482-8.

31. Jayawardene W, Dickinson S, Lohrmann D, Agley J. Arm circumference-to-height ratio as a situational alternative to BMI percentile in assessing obesity and cardiometabolic risk in adolescents. J Obes 2018;2018. https://doi.org/10.1155/2018/ 7456461.

32. Rerksuppaphol S, Rerksuppaphol L. Mid-upper-arm circumference and arm-to-height ratio to identify obesity in school-age children. Clin Med Res 2017:15:53-8.

33. Lu Q, Wang R, Lou DH, Ma CM, Liu XL, Yin FZ. Mid-upper-arm circumference and arm-to-height ratio in evaluation of overweight and obesity in Han children. Pediatr Neonatol 2014; 55:14-9.

34. Dean AG, Sullivan KM, Soe MM. Open source epidemiologic statistics for public health, version. www.OpenEpi.com updated 2013/04/06, [Accessed 11 Aug 2020].

35. WHO. WHO AnthroPlus software: software for assessing growth and development of the world's children. Geneva: WHO; 2007.

36. Flynn JT, Kaelber DC, Baker-Smith CM, Blowey D, Carroll AE, Daniels SR, et al. Clinical practice guideline for screening and management of high blood pressure in children and adolescents. Pediatrics 2017;140:e20171904.

37. Manios Y, Karatzi K, Protogerou AD, Moschonis G, Tsirimiagou C, Androutsos $\mathrm{O}$, et al. Prevalence of childhood hypertension and hypertension phenotypes by weight status and waist circumference: the healthy growth study. Eur J Nutr 2018;57:1147-55.

38. Wang H, Story RE, Venners SA, Wang B, Yang J, Li Z, et al. Patterns and interrelationships of body-fat measures among rural Chinese children aged 6 to 18 years. Pediatrics 2007;120:e94-101.

39. Shaw NJ, Crabtree NJ, Kibirige MS, Fordham JN. Ethnic and gender differences in body fat in British schoolchildren as measured by DXA. Arch Dis Child 2007;92:872-5.

40. Derman O, Yalçın SS, Kanbur NÖ, Kınık E. The importance of the measurement of the circumference of arm, arm muscle area and skinfold thickness during puberty. Int J Adolesc Med Health 2002;14: 193-7.

41. Yalçın SS, Kınık E. The impact of the sexual maturation stage on body mass index in adolescent girls. Turk J Pediatr 1999;11:315-21.

42. Barlow SE, Dietz WH. Obesity evaluation and treatment: expert committee recommendations. The maternal and child health bureau, health resources and services administration and the department of health and human services. Pediatrics 1998;102:E29.

43. Zhang Y-X, Wang M, Chu Z-H, Xie L. Prevalence of relatively high blood pressure among children and adolescents with different body mass index and subcutaneous fat cut-offs. Int J Cardiol 2015;179:536-8.

Supplementary Material: The online version of this article offers supplementary material (https://doi.org/10.1515/jpem-2020-0175). 